\title{
mammaNetz: Eine Begleitstelle für Frauen mit Brustkrebs in Augsburg
}

\author{
Andrea Thorenz Horst Erhardt \\ beta Institut für angewandtes Gesundheitsmanagement gGmbH, Augsburg, Deutschland
}

Eine bedarfsgerechte sektorenübergreifende Versorgung [1] kann in Deutschland nach unseren Erfahrungen besonders schwer für Patientinnen mit Brustkrebs sichergestellt werden, da sie langwierige und komplexe Behandlungsmaßnahmen benötigen. Sie durchlaufen mehrere Therapiephasen während ihrer Erkrankung und wechseln häufig zwischen den Sektoren (Fachbereiche Gynäkologie, Radiologie, Pathologie, Onkologie und Psychoonkologie). Ebenso häufig ist auch sozialmedizinische bzw. sozialberaterische Expertise gefragt. Darüber hinaus sind die Patientinnen krankheits- und behandlungsbedingten Belastungen ausgesetzt (z.B. körperliche Beschwerden wie Schmerzen, Einschränkung der Leistungsfähigkeit im Alltag, existentielle Bedrohung). Diese Belastungen sind häufig mit einer eingeschränkten Lebensqualität und psychischen Beeinträchtigung verbunden. So konnten internationale und nationale Studien zeigen, dass ein großer Teil der Frauen Angst vor dem Fortschreiten der Krankheit und dem Tod haben oder sich die Erkrankung negativ auf das Familien- und Berufsleben auswirkt [2-7]. Des Weiteren stellen die Studien Probleme im Informationsfluss und der Kommunikation zwischen den Behandlern und der Patientin sowie Defizite in der Vernetzung (gerade auch bei psychosozialen Therapiemaßnahmen) fest [8-10]. Diese Versorgungsmängel zu beheben, dafür setzt sich eine Case-Management-Begleitstelle für Frauen mit Brustkrebs ein.

\section{mammaNetz - Case-Management-Begleitstelle für Frauen mit Brustkrebs}

mammaNetz Augsburg ist die erste Begleit- und Orientierungsstelle für Frauen mit Brustkrebs in Deutschland, die Betroffene nach der Case-Management-Methode sektorenübergreifend von der Diagnose bis zur Nachsorge begleitet, berät und informiert. An mammaNetz kann sich jede Brustkrebspa- tientin aus der Region Augsburg wenden. Schon ab Diagnosestellung ist eine konstante Ansprechpartnerin (Case Managerin) wichtig, die sowohl die medizinischen Fakten vermittelt, als auch fachliche und emotionale Unterstützung leistet. Die Patientin steht mit ihren individuellen Sorgen und Problemen im Mittelpunkt der Begleitung. Aber auch Patientinnen in späteren Krankheitsphasen (z.B. bei Rezidiv, Metastasierung usw.) werden von mammaNetz betreut. Eine besonders wichtige Zielgruppe stellen psychisch und somatisch hoch belasteten Patientinnen dar. Die Spannweite des Angebots von mammaNetz reicht dabei von intensiver Begleitung bis zur «bloßen» Informationsgabe. Weitere Aufgaben von mammaNetz sind präventive Angebote, wie beispielsweise Brustselbstuntersuchungskurse. Bei mammaNetz werden jährlich zirka 300 Patientinnen betreut, von denen ein Drittel die Beratung (in der Regel 1-3 Kontakte) nutzen und zwei Drittel eine Case-Management-Begleitung erhalten. Ziel der Begleitstelle ist es, den Frauen (im Sinne von Case Management) eine Hilfe zur Selbsthilfe anzubieten und sie zu befähigen, sicherer und kompetenter mit der Erkrankung umzugehen. Zur Unterstützung einer kontinuierlichen, sektorenübergreifenden und patientenorientierten Begleitung werden die Patientinnen auf einem dafür neu entwickelten, strukturierten $\mathrm{Pa}-$ tientenpfad (Abb. 1) durch die verschiedenen Behandlungsphasen individuell begleitet.

Dieser Pfad wird unter Zuhilfenahme eines dafür entwickelten softwaregestützten Tools (Case-Management-Assisstent, erstellt in Zusammenarbeit mit Prof. Dr. Axel Tuma, Universität Augsburg) für jede Patientin abgebildet. Inhalt ist hier auch die Eingabe der Case-Management-Dokumente pro Patientin. Die Begleitung umfasst die Prozessschritte Intake, Assessment, Hilfeplan, Intervention und Bewertung und wurde in Anlehnung an die von der WHO [11, 12] aufgestellte ICF-Kategorisierung für multiaxiale Assessmentverfahren entwickelt [13].

\begin{tabular}{|c|c|}
\hline KARGER & (C) 2007 S. Karger GmbH, Freiburg \\
\hline $\begin{array}{l}\text { Fax +497614520714 } \\
\text { E-mail Information@Karger.de } \\
\text { www.karger.com }\end{array}$ & $\begin{array}{l}\text { Accessible online at: } \\
\text { www.karger.com/brc }\end{array}$ \\
\hline
\end{tabular}


Abb 1. Patientenpfad mammaNetz.

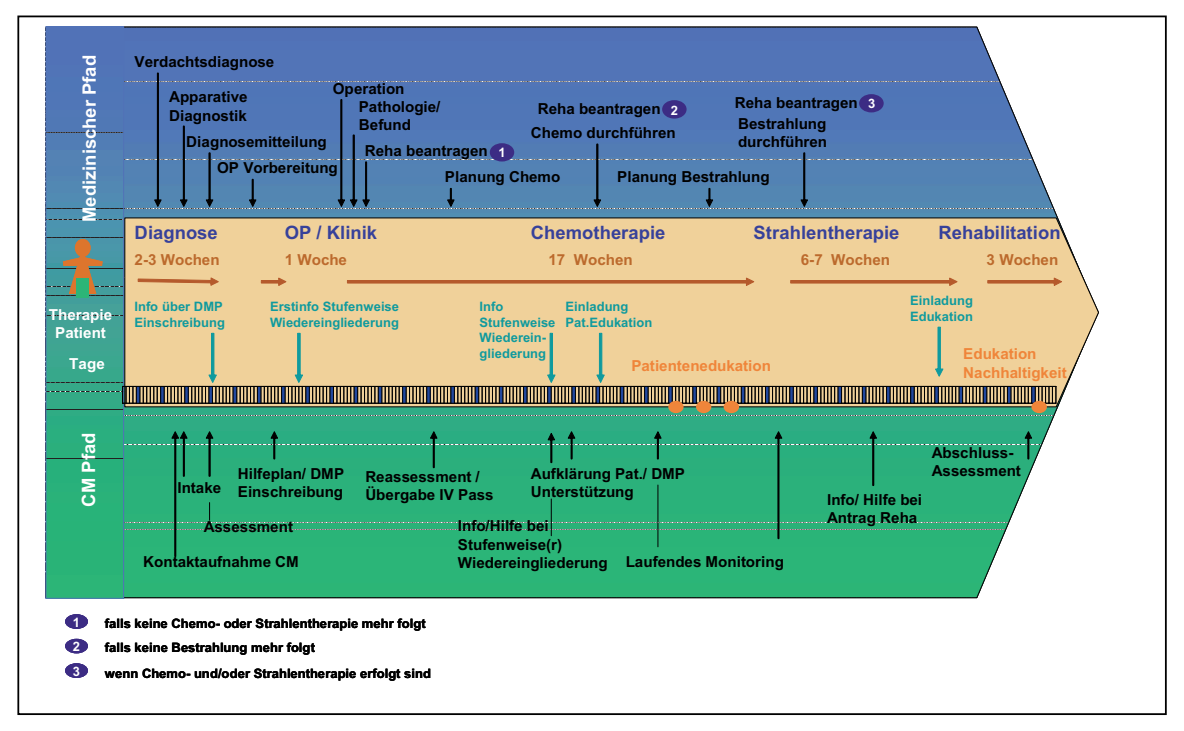

Das mammaNetz hat eine multiprofessionelle Vernetzung zwischen ambulantem, stationärem sowie Akut-, Reha- und Gemeinwesenbereich aufgebaut und sich mit dem Brustzentrum Augsburg, den Kliniken der Region und mit rund 50 gynäkologischen Praxen vernetzt, die kooperativ und qualitätsgesichert beteiligt sind. Darüber hinaus hat das mammaNetz ein Netzwerk mit rund 200 Einrichtungen, die brustkrebskranken Frauen helfen, etabliert.

Die Qualitätssicherung erfolgt nach anerkannten wissenschaftlichen Standards. Unter Zuhilfenahme der Methode des Care-Service-Science werden die Prozesse des mammaNetzes in zeitlichen Abständen überprüft und evaluiert und in einem Optimierungsverfahren im Anschluss so verbessert, dass man zu qualitätsgesicherten und praxiserprobten Ergebnissen gelangt (z.B. durch interdisziplinäre Fallbesprechungen). Die Begleitforschung [14] konnte bisher zeigen, dass das mammaNetz die Ängste vor den Auswirkungen der Erkrankung senkt und die Kompetenz im Umgang mit der Erkrankung erhöht. Die Studienpatientinnen zeigten eine hohe Akzeptanz und waren mit der Betreuung sehr zufrieden. Darüber hinaus wirkt sich die Organisation und der Ablauf der Behandlung und der Nachbetreuung positiv auf die Patientinnen aus. Die Netzwerkpartner (wie z.B. Gynäkologen, Onkologen usw.) beobachteten positive Auswirkungen der mammaNetz-Betreuung auf die Patientinnen.

\section{Finanzierung}

Projektträger dieser seit 2003 bestehenden Begleitstelle ist das beta Institut für angewandtes Gesundheitsmanagement in Augsburg. Das interdisziplinäre Team umfasst 10 Mitarbeiterinnen, darunter 5 vom beta Institut ausgebildete und zertifizierte Case Managerinnen. Nach einer Anschubfinanzierung von der betapharm Arzneimittel GmbH über ein dreiviertel Jahr wurde die Implementierung der Begleitstelle und eine Machbarkeitsstudie durch das Bayerische Staatsministerium innerhalb der Gesundheitsinitiative «Bayern aktiv» mit 1,25 Mio. Euro über drei Jahre Laufzeit (von Herbst 2002 bis 2005) finanziert. Seit Herbst 2005 finanziert sie sich über Fallpauschalen mit derzeit 23 Krankenkassen, die im Rahmen der Integrierten Versorgung nach $\S 140$ a SGB V vergütet werden. Soweit die Patientinnen bei diesen regionalen Krankenkassen versichert sind, erhalten sie die Begleitung kostenlos bzw. die Case Managerinnen stellen bei den anderen Krankenkassen jeweils Einzelfallanträge. Darüber hinaus besteht eine Förderung für bedürftige Frauen, die wenig verdienen und die entweder nicht krankenversichert sind oder deren Krankenkasse die Begleitung nicht übernimmt, durch den Verein zur Heilung von Brustkrebs, KOMEN Deutschland e.V.

Weitere Informationen unter www.mammaNetz.de.

\section{Literaturverzeichnis}

1 Sachverständigenrat für die konzertierte Aktion im Gesundheitswesen (SVR KAG): Bedarfsgerechtigkeit und Wirtschaftlichkeit. Band III.1: Über-, Unter- und Fehlversorgung, Gutachten 2000/2001. Nomos, Baden-Baden und: Koordination und Qualität im Gesundheitswesen, Gutachten 2005, Bundestags-Drucksache 15-5670.
2 Veronesi U, von Kleist S, Redmond K, Costa A, Delvaux N, Freilich G, Glaus A, Hudson T, McVie JG, Macnamara C, Meunier F, Pecorelli S, Serin D and the CAWAC Study Group: Caring about women and cancer (CAWAC): a European survey of the perspectives and experiences of women with female cancers. Eur J Cancer 1999;35:1667-1675.
3 Kaufmann M, Ernst B: Was Frauen mit Krebs erfahren, empfinden, wissen und vermissen. Dt Ärztebl 2000;97:A3191-3196.

4 Herschbach P, Keller M, Knight L, Brandl T, Huber B, Henrich G, Marten-Mittag, B: Psychological problems of cancer patients: a cancer screening with a cancer-specific questionnaire. Br J Cancer 2004;91: 504-511. 
5 Faller H: Krebs; in Jerusalem M, Weber H (Hrsg) Psychologische Gesundheitsförderung. Diagnostik und Prävention. Göttingen, Hogrefe, 2003, pp 655-675.

6 Goodwin PJ, Ennis M, Bordeleau LJ, Pritchard KI, Trudeau ME, Koo J, Hood, N: Health-related quality of life and psychosocial status in breast cancer prognosis: analysis of multiple variables. J Clin Oncol 2004;22:4184-4191.

7 Koch U, Weis J: Krankheitsbewältigung bei Krebs und Möglichkeiten der Unterstützung. Stuttgart, Schattauer, 1998, pp 59 ff.

8 Jänel G, Sing D, Grochocka A, Pfaff AB: «Es fehlt an Information und Kommunikation!» Probleme im Versorgungsprozess aus Sicht von Brustkrebspatientinnen und Ärzten. Forum DKG 2000;15:24-27.
9 Pfaff AB, Jänel G, Deutmeyer M, Grochocka A, Sing D, Schäfer-Walkmann S: Begleitforschung zur Qualitätssicherung an der Schnittstelle von ambulanter und stationärer Versorgung krebskranker Menschen - ambulant/stationäre Versorgungsqualität von Frauen mit Brustkrebs, Endbericht, Augsburg, 2001.

10 Kerr J, Engel J, Schlesinger-Raab A, Sauer H, Hölzel D: Communication, quality of life and age: results of a 5-year prospective study in breast cancer patients. Ann Oncol 2003;14:421-427.

11 WHO: Internationale Klassifikation der Funktionsfähigkeit, Behinderung und Gesundheit (ICF), WHO Publication, Genf, 2001
12 Stucki G, Grimby G: ICF Core sets for chronic conditions. J Rehabilitation 2004;44:205-210.

13 Thorenz A, Grochocka A: Case Management für Frauen mit Brustkrebs: Das mammaNetz Augsburg; in Case Management. Heidelberg, Economica, 2006, pp 81-85.

14 Thorenz A: Case Management zur Optimierung der sektorenübergreifenden Versorgung im Gesundheitswesen. Eine empirische Studie der Versorgung von Frauen mit Mammakarzinom durch das mammaNetz. Frankfurt/M., Peter Lang, 2007. 